\title{
Aerodynamic damping and fluid-structure interaction of blast loaded flexible structures
}

\author{
M. Teich \& N. Gebbeken \\ University of the German Armed Forces, Munich Campus, Germany
}

\begin{abstract}
This paper analyses the effects of air-structure interaction and derives a new coupling model for systems subjected to weak blast loads. While the coupling effects are negligible for typical steel or concrete structures, they may dominate the dynamic response of lighter and more flexible systems like membranes, blast curtains or cable facades. For these systems, a classical decoupled analysis, i.e., neglecting the influence of the surrounding air, might significantly overestimate the deflections and strains. The results of parameter studies are presented, and recommendations for the blast resistant design of flexible protective structures are given.
\end{abstract}

Keywords: aerodynamic damping, blast-structure interaction, flexible protective structure.

\section{Introduction}

The military has extensive knowledge and experience in protective design against blast and weapon effects. With increasing terrorist threats this knowledge is transferred to civilian applications [1]. Military protective structures like bunkers are often build with highly reinforced concrete. At the same time, civilian architectural trends head towards transparent, light and flexible structures like large cable net facades or membranes. Military methods and procedures, e.g. [2, 3], which are valid for the blast analysis of heavy and massive structures cannot be used in these cases. This paper contributes to a better understanding of the interaction of blast waves with light and compliant systems.

In the following Section 2, aerodynamic damping effects are illustrated. Then, in Section 3, we derive an analytical single degree of freedom (SDOF) fluid-structure interaction (FSI) model valid for the analysis of weak blast waves with linear or 
nonlinear structures. In Section 4, some parameter studies show the influence of aerodynamic damping and FSI on deflections and reflected pressure waves. Section 5 summarizes the main points of this paper and gives an outlook into further areas of research.

\section{Aerodynamic damping}

Aerodynamic damping is the result of energy dissipation of the surrounding air. While this effect is very small and usually neglected in standard protective design, it is taken into account for the design of structures subjected to strong wind loads and for flow-induced vibration analysis [4].

The basic principle of aerodynamic damping is illustrated in Figure 1. The plate with the specific mass $m$ and the velocity $\dot{x}$ impacts the air behind the system. This results in the propagation of an air wave moving with sound speed $c$ to the right. In the time span $\Delta t$ the wave travels the distance $\Delta x=c \Delta t$. The condition of continuity requires that the particle velocity $u_{p}$ is equal to $\dot{x}$. Thus, the affected air has the specific mass $m_{\text {air }}=\rho \Delta x=\rho c \Delta t$ and the momentum $\Delta i=m_{\text {air }} u_{p}=m_{\text {air }} \dot{x}=\rho c \Delta t \dot{x}$. For $\Delta t \rightarrow 0$, the air pressure corresponds to $\Delta i / \Delta t=\rho c \dot{x}$. However, this is a linear approximation assuming that the particle velocity $u_{p}$ is constant in the time span $\Delta t$ and neglecting complex reflection phenomena and pressure relief effects at building edges and corners [5]. To take account of these effects, an experimentally determined drag coefficient $c_{D}$ is included in the determination of the aerodynamic damping pressure,

$$
d_{a}=c_{D} \rho c \dot{x}
$$

which can also be written as

$$
d_{a}=2 m \omega \zeta_{a} \dot{x}
$$

for linear systems following classical structural damping notation. In this case, $\zeta_{a}$ denotes the aerodynamic damping ratio depending on the air properties density

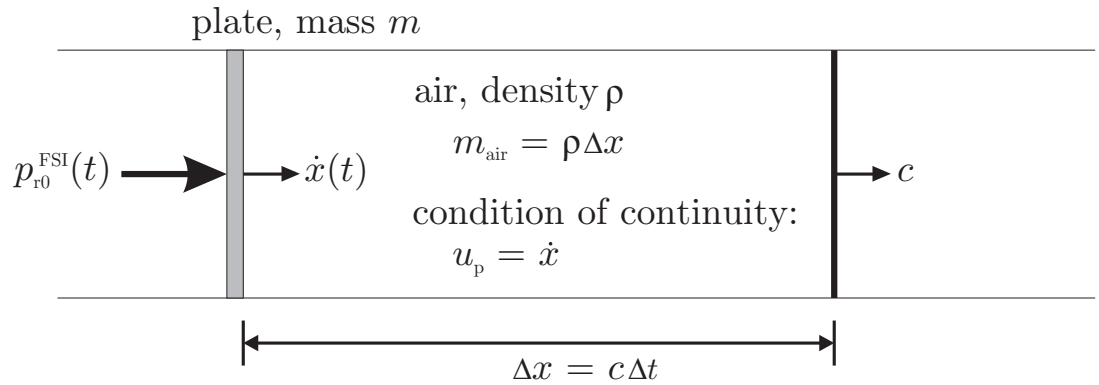

Figure 1: Basic principle of aerodynamic damping. 


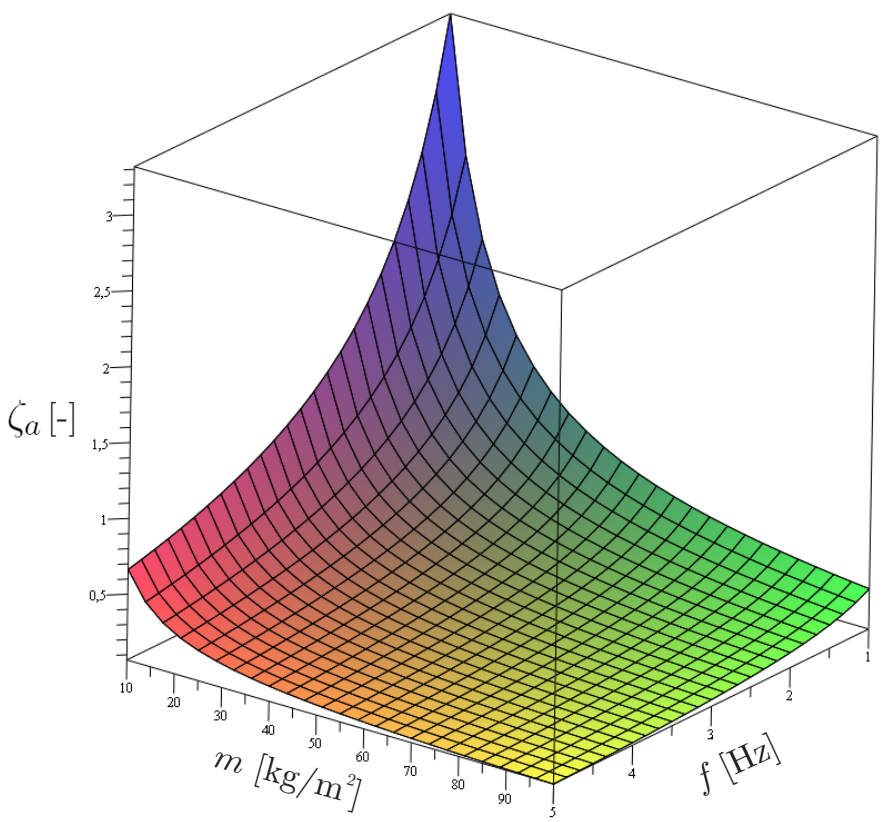

Figure 2: Aerodynamic damping ratio $\zeta_{a}$ for $c_{D}=1$ vs. mass $m$ and eigen frequency $f$.

and sound speed and on the structural properties mass and eigen frequency,

$$
\zeta_{a}=\frac{c_{D} \rho c}{2 m \omega}=\frac{c_{D} \rho c}{4 \pi f m} .
$$

Figure 2 illustrates the aerodynamic damping ratio $\zeta_{a}$ over specific mass and eigen frequency. For normal reinforced concrete and steel structures, the aerodynamic damping ratio is negligibly small. However, with decreasing mass and eigen frequency, the aerodynamic damping ratio increases significantly and reaches values up to $300 \%$ for very light and flexible systems (e.g. membrane structures, facades or blast curtains) compared to heavy rigid structures.

It may be of interest that this approach is also implemented in the European standard Eurocode 1, part 7, for the dynamic analysis of structures subjected to wind loads. In the Eurocode 1, the specific logarithmic damping decrement is defined as

$$
\Lambda_{a}=\frac{c_{D} \rho}{2 f m} v_{m}
$$

where $\Lambda_{a}=2 \pi \zeta_{a}$, and $v_{m}$ is the average wind velocity. 


\section{Analytical FSI model (TG-model)}

This section briefly describes the incident and reflected blast waves. Then, a new analytical FSI model (TG-model) is derived which is valid for the analysis of structures subjected to low-level blast loads.

\subsection{Incident blast wave}

Generally, the incident blast wave overpressure history $p_{10}(t)$ can be modeled with

$$
p_{10}(t)=\hat{p}_{10} \varphi(t)
$$

where $\hat{p}_{10}$ is the incident peak overpressure, and $\varphi(t)$ is a shape function describing the decay behaviour. Often, a simple triangular shape function is used, but recent studies $[6,7]$ have shown that the negative phase can significantly influence the structural response of flexible systems subjected to air blast loads.

Considering the negative phase, the Friedlander approach [8, 9]

$$
\varphi(t)=\left(1-\frac{t}{t_{d}}\right) \mathrm{e}^{-\alpha \frac{t}{t_{d}}}
$$

should be used as shape function for the incident blast wave $p_{10}(t)$. To accurately model the negative phase, the shape parameter $\alpha$ can be determined with

$$
\alpha=1.5 Z^{-0.38} \quad \text { for } 0.1<Z<30\left[\mathrm{~m} / \mathrm{kg}^{2}\right]
$$

based on the studies conducted by Borgers and Vantomme [10] where $Z=$ $R / M_{T N T}^{1 / 3}$ is the scaled detonation distance [11]. In comparison to other formula for the shape parameter, e.g. $[3,11]$, this approach reproduces the overpressure and especially the underpressure phase more accurately.

\subsection{Reflected blast wave}

When a blast wave hits a structure, it is reflected, and the reflected pressure acts on the structure. Generally, coupling effects are neglected, and the reflected pressuretime variation can then be determined with standard procedures, e.g. $[2,3,11]$. However, the deflection of the structure might influence the reflected pressure as shown in [12]. Due to the nonlinear characteristics of shock waves, general analytical solutions considering these coupling effects are not available. However, for the limit case of low-level blast loads, the derivation of an analytical solution is possible (Section 3.3).

The reflection coefficient $c_{r}$ describes the ratio of the peak reflected and the peak incident overpressure [13],

$$
c_{r}=\frac{\hat{p}_{r 0}}{\hat{p}_{10}}=\frac{8 \hat{p}_{10}+14 p_{0}}{\hat{p}_{10}+7 p_{0}} .
$$

For the limit of a very weak shock wave $c_{r}$ is close to 2 as illustrated in Figure 3. Figure 3 also shows the often used threat levels GSA C and GSA D according 


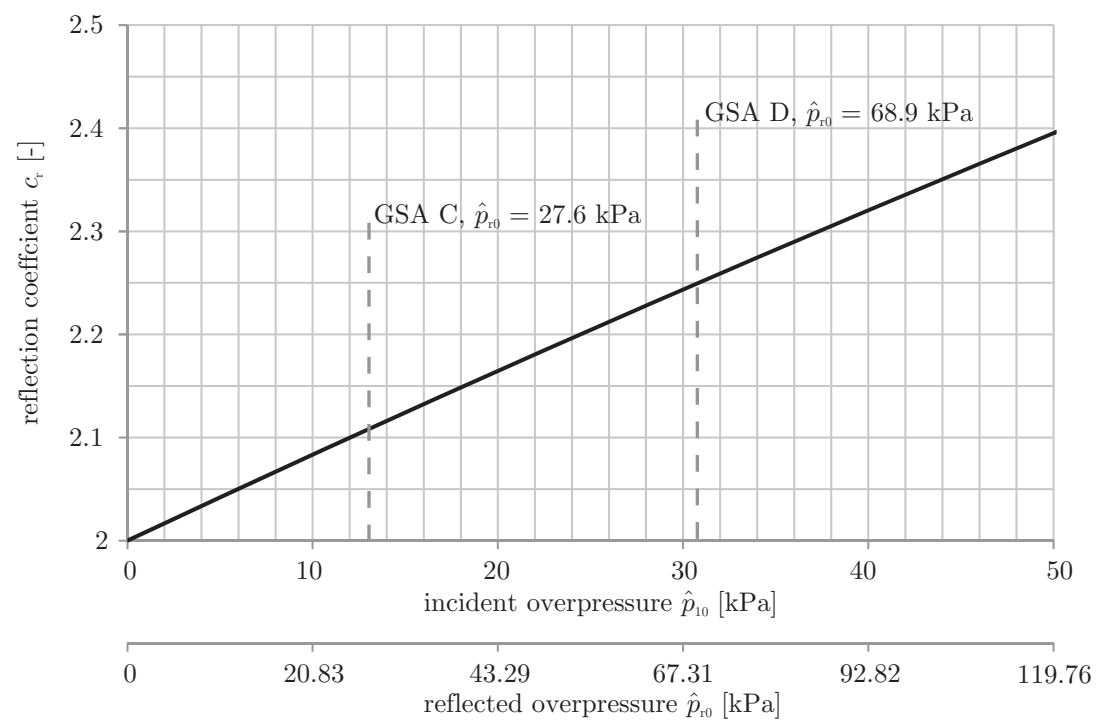

Figure 3: Reflection coefficient $c_{r}$ vs. incident overpressure $\hat{p}_{10}$.

to the U.S. General Services Administration (GSA) standard [14]. GSA level C is a far field threat scenario with a reflected peak overpressure of $27.6 \mathrm{kPa}$ and a reflected impulse of 193.1 Pas. This corresponds to a peak incident overpressure of $13.1 \mathrm{kPa}$ and a reflection coefficient of approx. 2.1. This is a typical low-level blast load.

For weak shock waves the conservation equations of mass, momentum and energy can be transformed into a linear wave equation [12, 13]. For this linear model, the principle of superposition is valid, and the reflected pressure history acting on a structure reads

$$
p_{r 0}^{\mathrm{FSI}}(t)=\hat{p}_{10}[\varphi(t)+\phi(t)]
$$

where $\phi(t)$ is the unknown shape function of the reflected wave, and the index FSI indicates the influence of FSI effects. Neglecting interaction effects yields $\phi(t)=\varphi(t)$, and the reflected pressure-time variation can be written as $p_{r 0}(t)=$ $c_{r} \hat{p}_{10} \varphi(t)$ with $c_{r} \approx 2$ for very weak shocks.

This linear approximation corresponds to the acoustic limit and is based on two main assumptions: (1) The speed of the blast wave is close to sound speed. (2) The medium transporting the blast wave is almost incompressible. These two assumptions are valid for the propagation of weak and medium shock waves in water. For air blast waves, however, the assumptions are only valid for low-level air blasts with small overpressures and a reflection coefficient of approximately 2 . 


\subsection{Equation of motion}

As illustrated in Figure 4, the equation of motion for a single degree of freedom (SDOF) model reads

$$
m \ddot{x}+d_{s}+d_{a}+r=p_{r 0}^{\mathrm{FSI}}=\hat{p}_{10}[\varphi+\phi]
$$

with the specific mass $m$, the structural damping pressure $d_{s}=d \dot{x}$, the aerodynamic damping pressure $d_{a}$ and the resistance function $r$ which depends on the deflection $x . p_{r 0}^{\mathrm{FSI}}$ is the reflected blast pressure. Both the displacement $x$ and the shape function $\phi$ are unknown. However, the condition of continuity requires that the structural velocity $\dot{x}$ needs to be equal to the sum of the wave velocities of the incident and of the reflected waves $[15,16]$. Applying the conservation of momentum, $p=\rho u c$, yields Taylor's FSI relation (see [12, 15] for more details)

$$
\dot{x}(t)=\frac{\hat{p}_{10}}{\rho c}[\varphi(t)-\phi(t)]
$$

or, equivalently,

$$
\phi(t)=\varphi(t)-\frac{\rho c}{\hat{p}_{10}} \dot{x} .
$$

This FSI relation couples the structural velocity $\dot{x}$ with the incident and the reflected wave shape functions $\varphi$ and $\phi . \rho$ is the density under ambient conditions (for air: $\rho=1.225 \mathrm{~kg} / \mathrm{m}^{3}$ ), and $c$ is the sound speed (for air: $c \approx 343 \mathrm{~m} / \mathrm{s}$ ).

With (12), the reflected pressure history (9) can be written as

$$
p_{r 0}^{\mathrm{FSI}}(t)=2 \hat{p}_{10} \varphi(t)-\frac{\rho c}{\hat{p}_{10}} \dot{x} .
$$

Thus the reflected pressure acting on a structure is altered depending on the structural velocity $\dot{x}$.

Combining (10) and (13) wields

$$
\ddot{x}+\left(\frac{d_{s}}{m}+c_{D} \frac{\rho c}{m}+\frac{\rho c}{m}\right) \dot{x}+\frac{r}{m}=\frac{2 \hat{p}_{10} \varphi}{m}
$$

or

$$
\ddot{x}+\left(\frac{d_{s}}{m}+c_{D} \frac{\rho c}{m}+\frac{\rho c}{m}\right) \dot{x}+\frac{r}{m}=\frac{c_{r} \hat{p}_{10} \varphi}{m}
$$

which is strictly only valid for $c_{r}=2.0$. However, we propose $c_{r} \approx 2.1$ as limit of application. The initial conditions are usually $x(0)=0$ and $\dot{x}(0)=0$.

The mass $m$ significantly influences the coupling effects. With increasing mass, $\rho c / m \rightarrow 0$ resulting in $p_{r 0}^{\mathrm{FSI}}(t)=2 \hat{p}_{10} \varphi(t)$ and $\phi(t)=\varphi(t)$. With decreasing mass and stiffness, however, coupling effects lead to a shape function $\phi(t)$ depending not only on time $t$ but also on the structural velocity. For very light systems, aerodynamic damping and the FSI term $\rho c / m$ dominate the solution of the differential equation (15) and the reflected pressure history $p_{r 0}^{\mathrm{FSI}}(t)(13)$. The vibration of the SDOF system is damped by three effects: (1) structural damping $d_{s}$, (2) aerodynamic damping $d_{a}$, and (3) damping due to FSI effects (term $\rho c / m$ ). However, only structural damping leads to internal forces in the system. 


\section{Examples}

To illustrate the coupling effects and their implication for practical design applications, we now turn to some numerical examples and analyze several systems with different eigen frequencies and masses subjected to a blast load as a result of the detonation of $100 \mathrm{~kg}$ TNT equivalent at a stand-off distance of $40 \mathrm{~m}$. This blast load corresponds approximately to the threat level $\mathrm{C}$ according to the U.S. GSA standard [14] and to the threat level EXV 33 according to the European standard [17]. The blast load parameters are summarized in Table 1. The incident peak pressure and incident impulse are computed with the formula of Kinney and Graham [11].

Generalized single degree of freedom (SDOF) models are a cost-effective alternative to powerful, but expensive, FE models. They are extremely useful for preliminary calculations and to check the results of more complex FE analyses. In contrast to FE modeling, they require very limited input data and are suitable for rapid analysis to get an "engineer feeling" for the structural performance. The approach to derive generalized SDOF models of real structures is well established in structural dynamics $[18,19]$ and is not repeated in this paper.

This section concentrates on a protective design in the linear elastic range where the resistance function reads $r=k x$. The circular eigen frequency is

Table 1: Blast load scenario: spherical detonation of $100 \mathrm{~kg}$ TNT equivalent at a stand-off distance of $40 \mathrm{~m}$.

\begin{tabular}{llll}
\hline & SYMBOL & VALUE & UNIT \\
\hline scaled distance & $Z$ & 8.62 & $\mathrm{~m} / \mathrm{kg}^{1 / 3}$ \\
\hline incident peak pressure & $\hat{p}_{10}$ & 12.2 & $\mathrm{kPa}$ \\
\hline incident impulse & $i^{+}$ & 113.1 & $\mathrm{kPa} \mathrm{ms}$ \\
\hline overpressure time duration & $t_{d}$ & 22.8 & $\mathrm{~ms}$ \\
\hline reflection coefficient & $c_{r}$ & 2.1 & - \\
\hline shape parameter & $\alpha$ & 0.66 & - \\
\hline
\end{tabular}

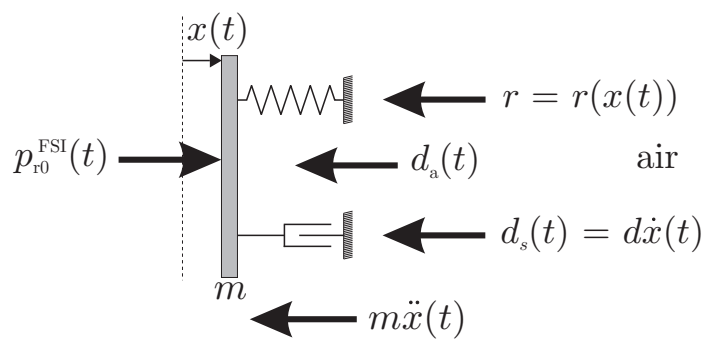

Figure 4: SDOF model: equilibrium of forces. 


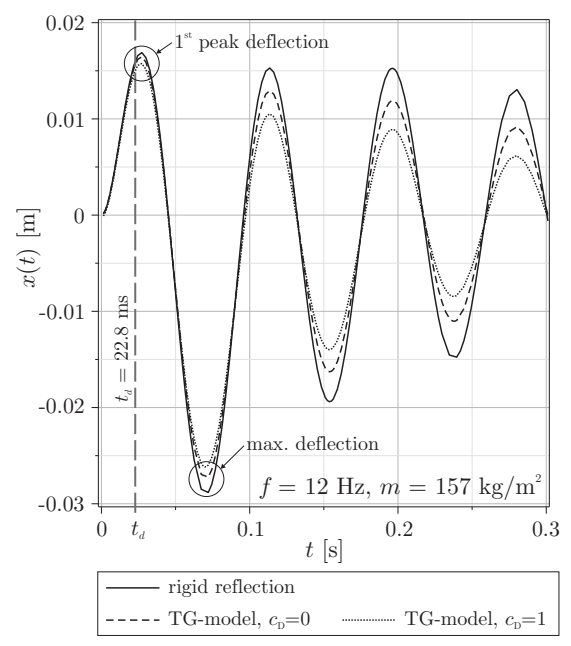

(a) $f=12 \mathrm{~Hz}, m=157 \mathrm{~kg} / \mathrm{m}^{2}$.

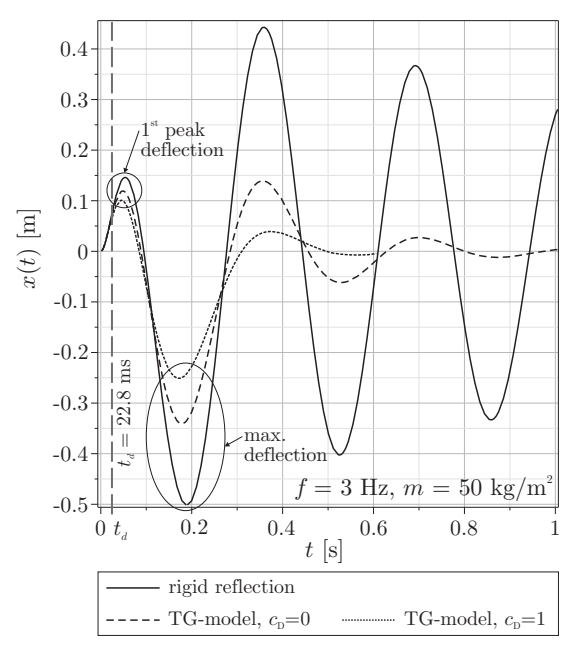

(b) $f=3 \mathrm{~Hz}, m=50 \mathrm{~kg} / \mathrm{m}^{2}$.

Figure 5: Displacements $x(t)$ over time for two systems with different eigen frequencies and different masses subjected to a detonation of $100 \mathrm{~kg}$ TNT equivalent at a stand-off distance of $40 \mathrm{~m}$ (3\% structural damping).

$\omega=\sqrt{k / m}=2 \pi f$ with the eigen frequency $f$. The differential equation reads

$$
\ddot{x}+\left(\frac{d_{s}}{m}+c_{D} \frac{\rho c}{m}+\frac{\rho c}{m}\right) \dot{x}+\omega^{2} x=\frac{c_{r} \hat{p}_{10} \varphi}{m}
$$

with structural damping $d_{s}=d \dot{x}=2 m \omega \zeta_{s} \dot{x}$.

In order to illustrate the basic effects of FSI and aerodynamic damping, Figure 5 shows the time dependant displacements of two systems. System 1 is moderately stiff ( $f=12 \mathrm{~Hz}$ ) with a specific mass of $m=157 \mathrm{~kg} / \mathrm{m}^{2}$. These properties correspond to a $2 \mathrm{~cm}$ thick, one-way steel plate with the dimensions $2 \mathrm{~m} \mathrm{x} 2 \mathrm{~m}$. The second system is more flexible $(f=3 \mathrm{~Hz})$ and has a specific mass of $m=50 \mathrm{~kg} / \mathrm{m}^{2}$ like typical facade and glazing systems.

Figure 5(a) compares the displacements of the first moderately stiff system when assuming rigid reflection (which is the typical approach in protective design) and when considering coupling effects based on the derived TG-model. The coupling effects are divided into two parts: (1) purely FSI without aerodynamic damping $\left(c_{D}=0\right)$ and (2) FSI and full aerodynamic damping $\left(c_{D}=1\right)$.

The first peak displacement of the moderately stiff and fully coupled system (dotted line in Figure 5(a)) is about 9\% smaller than the first peak displacement of the rigid analysis (solid line). As expected, the deformation attenuate quickest for the fully damped model. The deflection curves also underline the significance of the negative phase. For flexible systems, the negative phase usually determines the 
maximum deflection which is then directed contrary to the initial loading direction [7].

For the second and more flexible system shown in Figure 5(b), the coupling effects reduce the first peak displacement by approximately $40 \%$ and result in an even quicker attenuation of the time-dependant deformations. The coupling effects reduce the overpressure time duration of the reflected pressure history and the negative phase starts earlier. This contributes to the significant reduction of the first peak displacement.

All in all, the more flexible and the lighter the system is, the more significant are the coupling effects due to FSI and aerodynamic damping. There is a significant energy exchange between the fluid and the structural system.

The solutions for the shape functions $\phi(t)$ are shown in Figures 6 (a)-(b) for the flexible system with $f=3 \mathrm{~Hz}$ when (a) considering or (b) neglecting aerodynamic damping effects. Figures 6 (c)-(d) show the corresponding results for the stiffer system with $f=12 \mathrm{~Hz}$. Thus, Figures 6 (b) and (d) show the reflected wave profile $\phi(t)$ when purely considering FSI effects while Figures 6 (a) and (c) show $\phi(t)$ when taking account of FSI and full aerodynamic damping. The drag coefficient $c_{D}$ controls the amount of aerodynamic damping and is expected to be $0<c_{D}<1$ depending on shape and geometry. The value $c_{D}$ can only be obtained experimentally.

Figure 6 shows how the coupling effects due to FSI and aerodynamic damping influence the reflected wave profile $\phi(t)$ and thus the reflected pressure-time variation $p_{r 0}^{\mathrm{FSI}}(t)$. The smaller the mass, the smaller the overpressure time duration becomes. Thus, the positive impulse is also smaller for light structures. This is especially true for flexible systems with small eigen frequencies.

The coupling effects influence the negative phase, too. For the flexible system in Figure 6 (a), the negative impulse is also smaller than the impulse of the incident wave profile $\varphi(t)$. Without aerodynamic damping, there might be a second positive impulse after the negative phase as shown in Figure 6 (b) for the flexible system. Especially for very light systems, e.g. membrane systems or cable net facades, aerodynamic damping should be taken into account. For heavier systems with specific masses of $m \gtrsim 100 \mathrm{~kg} / \mathrm{m}^{2}$, the influence of aerodynamic damping is very small since $\zeta_{a} \rightarrow 0$.

Summarizing, we state that mass and stiffness influence the reflected wave profile $\phi(t)$. For light and flexible systems with low eigen frequencies, the overpressure time duration, the positive and the negative impulse decrease with decreasing mass. The influence becomes very significant for flexible systems with a specific mass $m \lesssim 50 \mathrm{~kg} / \mathrm{m}^{2}$. These light and flexible systems elude the applied blast load by undergoing large deflections.

\section{Summary and conclusions}

Relatively simple analytical models are important for the validation of complex numerical models and to give the engineer a feeling for the main parameters influencing the numerical results. 




(a) $f=3 \mathrm{~Hz}, c_{D}=1$.

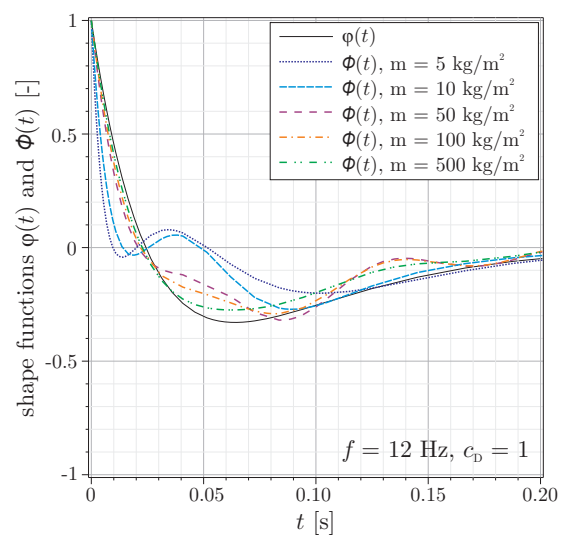

(c) $f=12 \mathrm{~Hz}, c_{D}=1$.

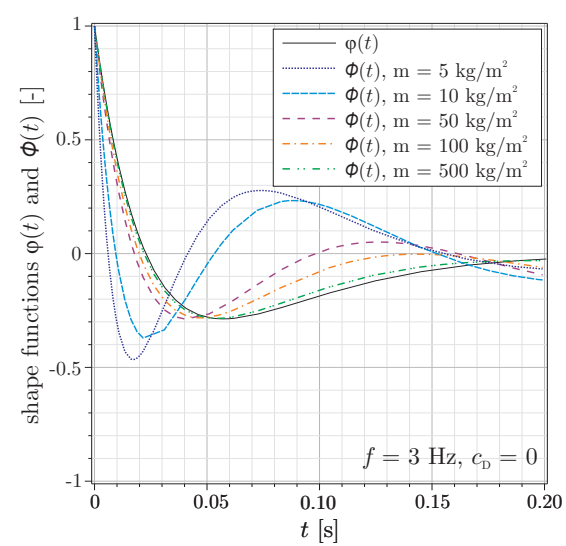

(b) $f=3 \mathrm{~Hz}, c_{D}=0$.



(d) $f=12 \mathrm{~Hz}, c_{D}=0$.

Figure 6: Shape functions over time of the incident and reflected waves, $\varphi(t)$ and $\phi(t)$, for different specific masses $m$ considering $\left(c_{D}=1\right)$ or neglecting $\left(c_{D}=0\right)$ aerodynamic damping. The blast scenario is a detonation of $100 \mathrm{~kg}$ TNT equivalent at a stand-off distance of $40 \mathrm{~m}$.

The derived linear TG-model is such a basic SDOF model and valid for the analysis of weak shocks with a reflection coefficient of approximately 2 . The authors propose $c_{r}<2.1$ as limit of application for the TG-model. This corresponds to a blast scenario with a scaled distance $Z>8.6 \mathrm{~kg} / \mathrm{m}^{1 / 3}$ and an incident peak overpressure $\hat{p}_{10}<12.3 \mathrm{kPa}$, respectively (approx. GSA threat level C [14], or European threat level ISO EXV 33 [17]). For stronger blast loads, compressibility effects become important which are not included in the presented TG-model. However, for low-amplitude shocks the TG-model might contribute to a deeper understanding of the physical coupling aspects.

The numerical studies highlight the effects of FSI and aerodynamic damping on 
the displacements and on the reflected shape function. For flexible systems with low eigen frequencies, the overpressure time duration, the positive and the negative impulse decrease with decreasing mass. The influence becomes very significant for flexible systems with a specific mass of $m<50 \mathrm{~kg} / \mathrm{m}^{2}$. The coupling effects largely influence the attenuation behavior.

\section{References}

[1] National Research Council (NRC), Protecting Buildings from Bomb Damage. Transfer of Blast-Effects Mitigation Technologies from Military to Civilian Applications. National Academy Press: Washington, D.C., 1995.

[2] US Army Corps of Engineers, TM 5-1300: Structures to Resist the Effects of Accidental Explosions, 1990.

[3] The Defence Special Weapons Agency and the Departments of the Army, Air Force, and Navy, TM 5-855-1: Design and Analysis of Hardened Strcutures to Conventional Weapons Effects, 1997. (for official use only).

[4] Vickery, B.J. \& Kao, K.H., Drag or along-wind response of slender structures. Journal of the Structural Division, 98, pp. 21-36, 1972.

[5] Rickman, D.D. \& Murrell, D.W., Development of an improved methodology for predicting airblast pressure relief on a directly loaded wall. Journal of Pressure Vessel Technology, 129(1), pp. 195-204, 2007.

[6] Krauthammer, T. \& Altenberg, A., Negative phase blast effects on glass panels. International Journal of Impact Engineering, 24(1), pp. 1-17, 2000.

[7] Teich, M. \& Gebbeken, N., The Influence of the Underpressure Phase on the Dynamic Response of Structures Subjected to Blast Loads. International Journal of Protective Structures, 1(2), pp. 219-233, 2010.

[8] Bulson, P., Explosive Loading of Engineering Structures. A History of Research and a Review of Recent Developments. E\&F Spon: London, 1997.

[9] Friedlander, F.G., Note on the diffraction of blast waves by a wall. UK Home Office Dept, RC(A), 1939.

[10] Borgers, J. \& Vantomme, J., Improving the accuracy of blast parameters using a new Friedlander curvature $\alpha$. DoD Explosives Safety Seminar, Palm Springs, CA, 2008.

[11] Kinney, G.F. \& Graham, K.J., Explosive Shocks in Air. Springer: New York, 1985.

[12] Teich, M. \& Gebbeken, N., Interaction of air blast waves with light and flexible structures. Design and Analysis of Protective Structures (DAPS), Singapore, 2010.

[13] Courant, R. \& Friedrichs, K.O., Supersonic Flow and Shock Waves, volume 21 of Applied Mathematical Sciences. Springer-Verlag: New York, 1976. (reprint of the edition of 1948).

[14] US General Services Administration, GSA-TS01-2003: Standard Test Method for Glazing and Window Systems, Subject to Dynamic Overpressure Loadings, 2003.

[15] Taylor, G.I., The pressure and impulse of submarine explosion waves 
on plates. The Scientific Papers of Sir Geoffrey Ingram Taylor, ed. G.K. Batchelor, Cambridge at the University Press: Cambridge, volume III Aerodynamics and the Mechanics of Projectiles and Explosions, chapter 31, pp. 287-303, 1963.

[16] Kriegsmann, G.A. \& Scandrett, C.L., Numerical studies of acoustic pulse scattering by baffled two-dimensional membranes. Journal of the Acoustical Society of America, 79(1), pp. 9-17, 1986.

[17] International Organization for Standardization, ISO 16933:2007: Glass in building - Explosion-resistant security glazing - Test and classification for arena air-blast loading, 2007.

[18] Biggs, J.M., Introduction to Structural Dynamics. McGraw-Hill: New York, 1964.

[19] Krauthammer, T., Modern Protective Structures. CRC, 2008. 